\title{
2 Accelerated Oxidation of Organic Micropollutants during $3 \quad$ Peracetic Acid Treatment in the Presence of Bromide Ion
}

5 a State Environmental Protection Key Laboratory of Integrated Surface Water-Groundwater

6 Pollution Control, School of Environmental Science and Engineering, Southern University of

$7 \quad$ Science and Technology, Shenzhen 518055, China

8 b Guangdong Provincial Key Laboratory of Soil and Groundwater Pollution Control, School of 9 Environmental Science and Engineering, Southern University of Science and Technology, 10 Shenzhen 518055, China

11 c The Key Laboratory of Water and Sediment Sciences, Ministry of Education, College of 12 Environmental Science and Engineering, Peking University, Beijing 100871, China

$13 *$ Corresponding author: J.J. Wang (Email: wangjj@sustech.edu.cn)

January 2022 
Text S1. Reactions Equations in OMPs degradation by the $\mathrm{PAA} / \mathrm{Br}^{-}$process.

$$
\begin{aligned}
& \frac{\mathrm{d}[\mathrm{PAA}]}{\mathrm{d} t}=-k_{\mathrm{R} 2}[\mathrm{PAAH}]\left[\mathrm{Br}^{-}\right]=-k_{\mathrm{R} 2}[\mathrm{PAA}]\left[\mathrm{Br}^{-}\right] \mathrm{g} \frac{\left[\mathrm{H}^{+}\right]}{\left[\mathrm{H}^{+}\right]+\mathrm{p} K_{\mathrm{a}, \mathrm{PAA}}} \\
& \frac{\mathrm{d}[\mathrm{HBrO}]}{\mathrm{d} t}=k_{\mathrm{R} 2}[\mathrm{PAAH}]\left[\mathrm{Br}^{-}\right]-k_{\mathrm{R} 5}[\mathrm{HBrO}][\mathrm{OMPH}]-k_{\mathrm{R} 6}[\mathrm{HBrO}]\left[\mathrm{OMP}^{-}\right]-k_{\mathrm{R} 8}\left[\mathrm{HBrO}_{[}\left[\mathrm{HO}_{2}^{-}\right]-k_{\mathrm{R} 9}\left[\mathrm{BrO}^{-}\right]\left[\mathrm{H}_{2} \mathrm{O}_{2}\right]\right. \\
& =-k_{\mathrm{R} 2}[\mathrm{PAA}]\left[\mathrm{Br}{ }^{-}\right] \mathrm{g} \frac{\left[\mathrm{H}^{+}\right]}{\left[\mathrm{H}^{+}\right]+\mathrm{p} K_{\mathrm{a}, \mathrm{PAA}}}-k_{\mathrm{R} 5}[\mathrm{HBrO}][\mathrm{OMP}] \mathrm{g} \frac{\left[\mathrm{H}^{+}\right]}{\left[\mathrm{H}^{+}\right]+\mathrm{p} K_{\mathrm{a}, \mathrm{HBrO}}} \mathrm{g} \frac{\left[\mathrm{H}^{+}\right]}{\left[\mathrm{H}^{+}\right]+\mathrm{p} K_{\mathrm{a}, \mathrm{OMP}}} \\
& -k_{\mathrm{R} 6}[\mathrm{HBrO}][\mathrm{OMP}] \mathrm{g} \frac{\left[\mathrm{H}^{+}\right]}{\left[\mathrm{H}^{+}\right]+\mathrm{p} K_{\mathrm{a}, \mathrm{HBrO}}} \mathrm{g} \frac{\mathrm{p} K_{\mathrm{a}, \mathrm{OMP}}}{\left[\mathrm{H}^{+}\right]+\mathrm{p} K_{\mathrm{a}, \mathrm{OMP}}}-k_{\mathrm{R} 8}[\mathrm{HBrO}]\left[\mathrm{H}_{2} \mathrm{O}_{2}\right] \mathrm{g} \frac{\left[\mathrm{H}^{+}\right]}{\left[\mathrm{H}^{+}\right]+\mathrm{p} K_{\mathrm{a}, \mathrm{HBrO}}} \mathrm{g} \frac{\mathrm{p} K_{\mathrm{a}, \mathrm{H}_{2} \mathrm{O}_{2}}}{\left[\mathrm{H}^{+}\right]+K_{\mathrm{a}, \mathrm{H}_{2} \mathrm{O}_{2}}} \\
& -k_{\mathrm{R} 9}[\mathrm{HBrO}]\left[\mathrm{H}_{2} \mathrm{O}_{2}\right] \mathrm{g} \frac{\mathrm{p} K_{\mathrm{a}, \mathrm{HBrO}}}{\left[\mathrm{H}^{+}\right]+\mathrm{p} K_{\mathrm{a}, \mathrm{HBrO}}} \mathrm{g} \frac{\left[\mathrm{H}^{+}\right]}{\left[\mathrm{H}^{+}\right]+\mathrm{p} K_{\mathrm{a}, \mathrm{H}_{2} \mathrm{O}_{2}}} \\
& \frac{\mathrm{d}[\mathrm{OMP}]}{\mathrm{d} t}=-k_{\mathrm{R} 5}[\mathrm{HBrO}][\mathrm{OMPH}]-k_{\mathrm{R} 6}[\mathrm{HBrO}]\left[\mathrm{OMP}^{-}\right] \\
& =-k_{\mathrm{R} 5}[\mathrm{HBrO}][\mathrm{OMP}] \mathrm{g} \frac{\left[\mathrm{H}^{+}\right]}{\left[\mathrm{H}^{+}\right]+\mathrm{p} K_{\mathrm{a}, \mathrm{HBrO}}} \mathrm{g} \frac{\left[\mathrm{H}^{+}\right]}{\left[\mathrm{H}^{+}\right]+\mathrm{p} K_{\mathrm{a}, \mathrm{OMP}}}-k_{\mathrm{R} 6}[\mathrm{HBrO}][\mathrm{OMP}] \mathrm{g} \frac{\left[\mathrm{H}^{+}\right]}{\left[\mathrm{H}^{+}\right]+\mathrm{p} K_{\mathrm{a}, \mathrm{HBrO}}} \mathrm{g} \frac{\mathrm{p} K_{\mathrm{a}, \mathrm{OMP}}}{\left[\mathrm{H}^{+}\right]+\mathrm{p} K_{\mathrm{a}, \mathrm{OMP}}}
\end{aligned}
$$




\section{Text S2. Toxicity Prediction}

USEPA ECOSAR v2.0 software was employed to predict the toxicity of target compounds and their reaction products. $\frac{1}{}$ By using computerized structure activity relationships, the ECOSAR can estimate the aquatic toxicity on the basis of structure similarity of a given compound with known toxicity values of other compounds. In this study, the acute (short-term) toxicity (LC50 and EC50) and chronic (long-term or delayed) toxicity (ChV) to green algae, daphnid, and fish were estimated. 
Table S1. Selected properties of ethinylestradiol (EE2), sulfamethoxazole (SMX), naproxen (NPX), phenol (PHE), peracetic acid (PAA), and hydrogen peroxide $\left(\mathrm{H}_{2} \mathrm{O}_{2}\right)$.

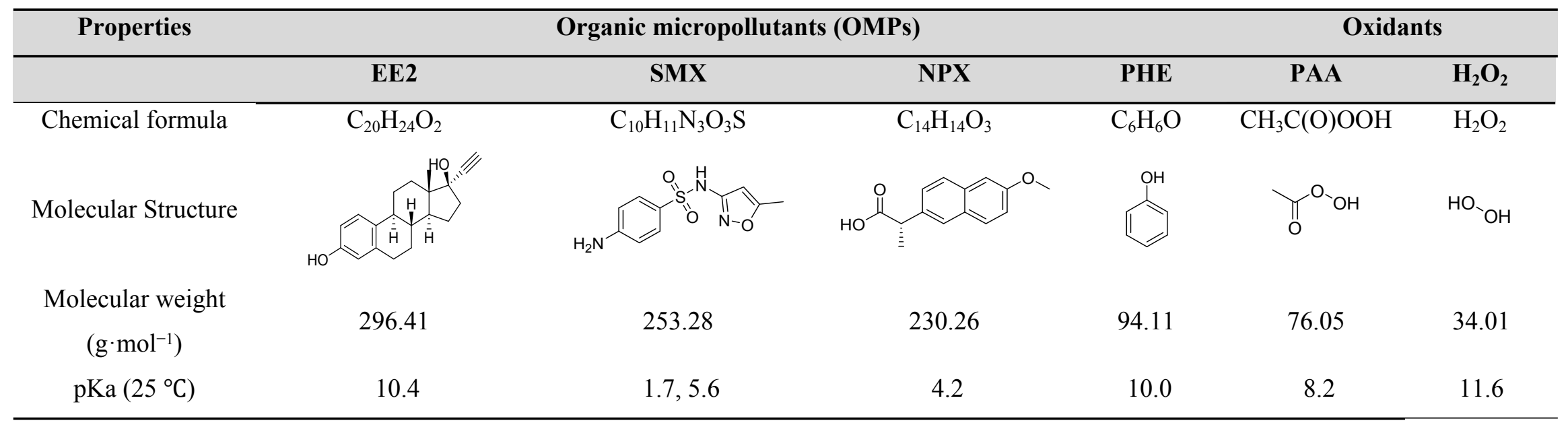


Table S2. Pseudo-first-order rate constants $\left(k_{\text {obs }}\right)$ of PAA self-decay or decay by $\mathrm{Br}^{-}$at pH 4.0-9.0 and initial $\left[\mathrm{H}_{2} \mathrm{O}_{2}\right]_{0}$ of $0.1-1.0 \mathrm{mM}^{-}$ (pH 7.1), and pH 4.0-8.0 ( $\left.\mathrm{R}^{2}>0.997\right)$. Reaction conditions unless specified: $[\mathrm{PAA}]_{0}=30 \mu \mathrm{M},\left[\mathrm{Br}^{-}\right]_{0}=15 \mathrm{mM},\left[\mathrm{H}_{2} \mathrm{O}_{2}\right]_{0}=15 \mu \mathrm{M}, \mathrm{pH}$ $=7.1$, and room temperature $\left(22 \pm 2^{\circ} \mathrm{C}\right)$.

\begin{tabular}{|c|c|c|c|c|c|}
\hline $\begin{array}{l}\text { PAA self- } \\
\text { decay at }\end{array}$ & $k_{\mathrm{obs}}\left(\mathrm{min}^{-1}\right)$ & $\begin{array}{c}\text { PAA decay } \\
\text { by } \mathrm{Br}^{-} \text {at }\end{array}$ & $k_{\mathrm{obs}}\left(\min ^{-1}\right)$ & $\begin{array}{c}\text { PAA decay by } \\
\mathrm{Br}^{-} \text {at }\end{array}$ & $k_{\text {obs }}\left(\min ^{-1}\right)$ \\
\hline pH 5.0 & $1.38 \pm 0.09 \times 10^{-4}$ & $\mathrm{pH} 4.0$ & $1.81 \pm 0.01 \times 10^{-1}$ & $\mathrm{H}_{2} \mathrm{O}_{2} 15 \mu \mathrm{M}$ & $1.70 \pm 0.01 \times 10^{-1}$ \\
\hline pH 6.0 & $2.22 \pm 0.10 \times 10^{-4}$ & pH 5.0 & $1.79 \pm 0.02 \times 10^{-1}$ & $\mathrm{H}_{2} \mathrm{O}_{2} 30 \mu \mathrm{M}$ & $1.77 \pm 0.01 \times 10^{-1}$ \\
\hline pH 7.1 & $1.00 \pm 0.04 \times 10^{-3}$ & pH 6.0 & $1.79 \pm 0.02 \times 10^{-1}$ & $\mathrm{H}_{2} \mathrm{O}_{2} 45 \mu \mathrm{M}$ & $1.78 \pm 0.01 \times 10^{-1}$ \\
\hline pH 8.0 & $1.32 \pm 0.05 \times 10^{-3}$ & pH 7.1 & $1.70 \pm 0.01 \times 10^{-1}$ & $\mathrm{H}_{2} \mathrm{O}_{2} 60 \mu \mathrm{M}$ & $1.85 \pm 0.02 \times 10^{-1}$ \\
\hline \multirow[t]{5}{*}{ pH 9.0} & $1.55 \pm 0.03 \times 10^{-3}$ & pH 7.4 & $1.46 \pm 0.03 \times 10^{-1}$ & $\mathrm{H}_{2} \mathrm{O}_{2} 150 \mu \mathrm{M}$ & $1.75 \pm 0.01 \times 10^{-1}$ \\
\hline & & $\mathrm{pH} 7.8$ & $1.35 \pm 0.02 \times 10^{-1}$ & & \\
\hline & & $\mathrm{pH} 8.2$ & $8.87 \pm 0.04 \times 10^{-2}$ & & \\
\hline & & pH 8.6 & $5.60 \pm 0.10 \times 10^{-2}$ & & \\
\hline & & pH 9.0 & $2.30 \pm 0.12 \times 10^{-2}$ & & \\
\hline
\end{tabular}


Table S3. List of the primary elementary reactions and the rate constants in $\mathrm{OMPs} / \mathrm{HBrO}$ system.

\begin{tabular}{llll}
\hline \multicolumn{2}{c}{ Reactions } & $\boldsymbol{k}\left(\mathbf{M}^{-1} \cdot \mathbf{s}^{-1}\right)$ & Reference \\
\hline SMX/HBrO reactions: & & \\
$\mathrm{R} 10$ & $\mathrm{SMX} \rightleftharpoons \mathrm{SMX}^{-}+\mathrm{H}^{+}$ & $1.26 \times 10^{5} \mathrm{~s}^{-1}, \mathrm{pKa}=5.6$ & \\
$\mathrm{R} 10 \mathrm{r}$ & & $5.0 \times 10^{10}$ & \\
$\mathrm{R} 11$ & $\mathrm{HBrO}+\mathrm{SMX} \rightarrow$ products & $1.1 \times 10^{5 \mathrm{a}}, \underline{3}$ \\
$\mathrm{R} 12$ & $\mathrm{HBrO}+\mathrm{SMX}^{-} \rightarrow$ products & $2.3 \times 10^{5 \mathrm{a}}$ & $\underline{2}, \underline{3}$ \\
NPX/HBrO reactions: & & \\
$\mathrm{R} 10$ & $\mathrm{NPX} \rightleftharpoons \mathrm{NPX}+\mathrm{H}^{+}$ & $3.15 \times 10^{6} \mathrm{~s}^{-1}, \mathrm{pKa}=4.2$ & \\
$\mathrm{R} 10 \mathrm{r}$ & & $5.0 \times 10^{10}$ & \\
$\mathrm{R} 11$ & $\mathrm{H}^{+}+\mathrm{HBrO}+\mathrm{NPX} \rightarrow$ products & $<1.0 \times 10^{8 \mathrm{~b}} \mathrm{M}^{-2} \cdot \mathrm{s}^{-1}$ & $\underline{4}$ \\
$\mathrm{R} 12$ & $\mathrm{HBrO}+\mathrm{NPX} \rightarrow$ products & $2.1 \times 10^{5 \mathrm{c}, \mathrm{d}}$ & $\underline{4}$ \\
$\mathrm{R} 13$ & $\mathrm{HBrO}+\mathrm{NPX} \rightarrow$ products & $2.7 \times 10^{2 \mathrm{~d}}$ & $\underline{4}$
\end{tabular}

\section{PHE/HBrO reactions:}

$\begin{array}{llll}\mathrm{R} 10 & \mathrm{PHE} \rightleftharpoons \mathrm{PHE}^{-}+\mathrm{H}^{+} & 5 \mathrm{~s}^{-1}, \mathrm{pKa}=10.0 & \\ \text { R10r } & & 5.0 \times 10^{10} & \\ \mathrm{R} 11 & \mathrm{HBrO}+\mathrm{PHE} \rightarrow \text { products } & 5.0 \times 10^{2 \mathrm{e}} & \underline{4-6} \\ \mathrm{R} 12 & \mathrm{HBrO}+\mathrm{PHE}^{-} \rightarrow \text { products } & 1.0 \times 10^{8 \mathrm{e}} & \underline{4-6}\end{array}$

${ }^{\mathrm{a}}$ Estimated from literature; ${ }^{\mathrm{b}}$ acid-catalyzed reaction mechanism; ${ }^{\mathrm{c}}$ original reported rate constant is $2.1 \times 10^{4} \mathrm{M}^{-1} \cdot \mathrm{s}^{-1}$, which should be $2.1 \times 10^{5} \mathrm{M}^{-1} \cdot \mathrm{s}^{-1}$ to fit their reported data correctly; ${ }^{\mathrm{d}}$ the reported data does not fit our model, especially under neutral $\mathrm{pH}$ conditions, $6.0 \times 10^{4} \mathrm{M}^{-1} \cdot \mathrm{s}^{-1}$ was estimated as $k_{\text {app }}$ of $\mathrm{HBrO}$ with NPX at $\mathrm{pH}$ 6.0-8.0 for better fitting results; e the reported experimental $k_{\text {app }}$ at specific $\mathrm{pH}$ in literature may also be used. 
Table S4. Pseudo-first-order rate constants $\left(k_{\mathrm{obs}}\right)$ of degradation of OMPs by the PAA/Br- system at initial $\left[\mathrm{Br}^{-}\right]_{0}$ of $0.05-0.4 \mathrm{mM}$,

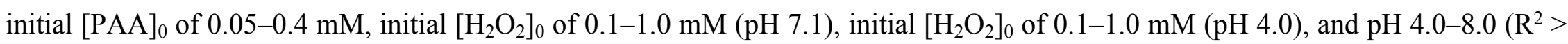
0.927). Reaction conditions unless specified: $[\mathrm{OMP}]_{0}=10 \mu \mathrm{M},\left[\mathrm{Br}^{-}\right]_{0}=0.1 \mathrm{mM},[\mathrm{PAA}]_{0}=0.2 \mathrm{mM},\left[\mathrm{H}_{2} \mathrm{O}_{2}\right]_{0}=0.2 \mathrm{mM}, \mathrm{pH}=7.1$, and room temperature $\left(22 \pm 2{ }^{\circ} \mathrm{C}\right)$.

\begin{tabular}{|c|c|c|c|c|c|}
\hline & & \multicolumn{4}{|c|}{$k_{\text {obs }}\left(\min ^{-1}\right)$} \\
\hline & & EE2 & SMX & NPX & PHE \\
\hline \multirow{2}{*}{$\begin{array}{l}\text { Initial } \\
{\left[\mathrm{Br}^{-}\right]_{0}}\end{array}$} & 0.05 & $2.78 \pm 0.09 \times 10^{-3}$ & $3.08 \pm 0.02 \times 10^{-3}$ & $1.06 \pm 0.04 \times 10^{-3}$ & $2.35 \pm 0.07 \times 10^{-3}$ \\
\hline & 0.1 & $6.56 \pm 0.21 \times 10^{-3}$ & $8.45 \pm 0.03 \times 10^{-3}$ & $3.24 \pm 0.03 \times 10^{-3}$ & $3.49 \pm 0.13 \times 10^{-3}$ \\
\hline \multirow[t]{2}{*}{$(\mathrm{mM})$} & 0.2 & $1.14 \pm 0.02 \times 10^{-2}$ & $2.65 \pm 0.02 \times 10^{-2}$ & $6.31 \pm 0.05 \times 10^{-3}$ & $6.75 \pm 0.23 \times 10^{-3}$ \\
\hline & 0.4 & $1.99 \pm 0.02 \times 10^{-2}$ & $9.02 \pm 0.37 \times 10^{-2}$ & $2.18 \pm 0.03 \times 10^{-2}$ & $1.25 \pm 0.05 \times 10^{-2}$ \\
\hline \multirow{2}{*}{$\begin{array}{l}\text { Initial } \\
{[\mathrm{PAA}]_{0}}\end{array}$} & 0.05 & $3.88 \pm 0.09 \times 10^{-3}$ & $6.72 \pm 0.06 \times 10^{-3}$ & $2.99 \pm 0.04 \times 10^{-3}$ & $2.54 \pm 0.06 \times 10^{-3}$ \\
\hline & 0.1 & $6.15 \pm 0.05 \times 10^{-3}$ & $1.32 \pm 0.01 \times 10^{-3}$ & $4.25 \pm 0.22 \times 10^{-3}$ & $5.13 \pm 0.14 \times 10^{-3}$ \\
\hline \multirow[t]{2}{*}{$(\mathrm{mM})$} & 0.2 & $1.14 \pm 0.02 \times 10^{-2}$ & $2.65 \pm 0.09 \times 10^{-2}$ & $6.31 \pm 0.05 \times 10^{-3}$ & $6.75 \pm 0.23 \times 10^{-3}$ \\
\hline & 0.4 & $1.86 \pm 0.02 \times 10^{-2}$ & $6.53 \pm 0.24 \times 10^{-2}$ & $1.32 \pm 0.02 \times 10^{-2}$ & $9.10 \pm 0.42 \times 10^{-3}$ \\
\hline Initial & 0.1 & $1.14 \pm 0.02 \times 10^{-2}$ & $2.65 \pm 0.09 \times 10^{-2}$ & $6.31 \pm 0.05 \times 10^{-3}$ & $6.75 \pm 0.23 \times 10^{-3}$ \\
\hline
\end{tabular}




\begin{tabular}{|c|c|c|c|c|c|}
\hline$\left[\mathrm{H}_{2} \mathrm{O}_{2}\right]_{0}$ at & 0.3 & $9.00 \pm 0.05 \times 10^{-3}$ & $1.43 \pm 0.01 \times 10^{-2}$ & $3.98 \pm 0.09 \times 10^{-3}$ & $3.51 \pm 0.14 \times 10^{-3}$ \\
\hline $\mathrm{pH} 7.1$ & 0.5 & $6.40 \pm 0.06 \times 10^{-3}$ & $9.57 \pm 0.18 \times 10^{-3}$ & $3.29 \pm 0.13 \times 10^{-3}$ & $1.67 \pm 0.07 \times 10^{-3}$ \\
\hline$(\mathrm{mM})$ & 1.0 & $3.56 \pm 0.07 \times 10^{-3}$ & $5.72 \pm 0.22 \times 10^{-3}$ & $2.28 \pm 0.11 \times 10^{-3}$ & $6.19 \pm 0.18 \times 10^{-4}$ \\
\hline Initial & 0.1 & $4.27 \pm 0.14 \times 10^{-2}$ & $6.43 \pm 0.65 \times 10^{-2}$ & $4.28 \pm 0.23 \times 10^{-1}$ & $3.09 \pm 0.12 \times 10^{-2}$ \\
\hline$\left[\mathrm{H}_{2} \mathrm{O}_{2}\right]_{0}$ at & 0.3 & $3.62 \pm 0.09 \times 10^{-2}$ & $7.46 \pm 0.90 \times 10^{-2}$ & $4.44 \pm 0.28 \times 10^{-1}$ & $1.76 \pm 0.01 \times 10^{-2}$ \\
\hline & 0.5 & $3.55 \pm 0.10 \times 10^{-2}$ & $7.61 \pm 0.82 \times 10^{-2}$ & $3.49 \pm 0.17 \times 10^{-2}$ & $1.23 \pm 0.01 \times 10^{-2}$ \\
\hline & 1.0 & $2.79 \pm 0.18 \times 10^{-2}$ & $5.96 \pm 0.31 \times 10^{-2}$ & $3.30 \pm 0.12 \times 10^{-2}$ & $6.90 \pm 0.17 \times 10^{-3}$ \\
\hline $\mathrm{PH}$ & 4.0 & $4.27 \pm 0.14 \times 10^{-2}$ & $6.92 \pm 0.86 \times 10^{-2}$ & $4.28 \pm 0.23 \times 10^{-1}$ & $3.09 \pm 0.12 \times 10^{-2}$ \\
\hline & 5.0 & $3.14 \pm 0.10 \times 10^{-2}$ & $6.62 \pm 0.80 \times 10^{-2}$ & $3.61 \pm 0.24 \times 10^{-2}$ & $1.58 \pm 0.03 \times 10^{-2}$ \\
\hline & 6.0 & $1.99 \pm 0.03 \times 10^{-2}$ & $5.96 \pm 0.65 \times 10^{-2}$ & $2.61 \pm 0.09 \times 10^{-2}$ & $9.00 \pm 0.36 \times 10^{-3}$ \\
\hline & 7.1 & $1.14 \pm 0.02 \times 10^{-2}$ & $2.65 \pm 0.09 \times 10^{-2}$ & $6.31 \pm 0.05 \times 10^{-3}$ & $6.75 \pm 0.23 \times 10^{-3}$ \\
\hline & 8.0 & $9.84 \pm 0.29 \times 10^{-3}$ & $1.45 \pm 0.15 \times 10^{-3}$ & $1.07 \pm 0.09 \times 10^{-3}$ & $6.57 \pm 0.30 \times 10^{-3}$ \\
\hline
\end{tabular}

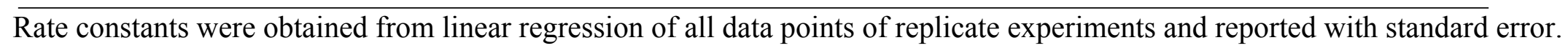


Table S5. HPLC/ESI-QqQMS data of the brominated products of OMPs in the PAA/Br- process at PIS mode of $m / z 79$.

\begin{tabular}{|c|c|c|c|}
\hline $\begin{array}{c}\text { Parent } \\
\text { compound }\end{array}$ & $\begin{array}{c}\text { Measured } \\
\text { mass } \\
(\mathrm{m} / \mathrm{z})\end{array}$ & $\begin{array}{c}\text { Molecular } \\
\text { formula }\end{array}$ & $\begin{array}{c}\text { Retention } \\
\text { time } \\
\text { (min) }\end{array}$ \\
\hline \multirow{7}{*}{ EE2 } & 373.1 & $\mathrm{C}_{20} \mathrm{H}_{23} \mathrm{O}_{2} \mathrm{Br}$ & $6.20 / 6.50$ \\
\hline & 389.1 & $\mathrm{C}_{20} \mathrm{H}_{23} \mathrm{O}_{3} \mathrm{Br}$ & $4.19 / 4.54$ \\
\hline & 435.1 & $\mathrm{C}_{20} \mathrm{H}_{22} \mathrm{OBr}_{2}$ & 5.62 \\
\hline & 451.0 & $\mathrm{C}_{20} \mathrm{H}_{22} \mathrm{O}_{2} \mathrm{Br}_{2}$ & 7.25 \\
\hline & 469.0 & $\mathrm{C}_{20} \mathrm{H}_{22} \mathrm{O}_{3} \mathrm{Br}_{2}$ & 4.98 \\
\hline & 512.9 & $\mathrm{C}_{20} \mathrm{H}_{21} \mathrm{OBr}_{3}$ & 6.08 \\
\hline & 546.9 & $\mathrm{C}_{20} \mathrm{H}_{21} \mathrm{O}_{3} \mathrm{Br}_{3}$ & 6.78 \\
\hline \multirow[t]{2}{*}{ SMX } & 332.0 & $\mathrm{C}_{10} \mathrm{H}_{10} \mathrm{~N}_{3} \mathrm{O}_{3} \mathrm{SBr}$ & $4.65 / 9.25$ \\
\hline & 307.0 & $\mathrm{C}_{14} \mathrm{H}_{14} \mathrm{O}_{3} \mathrm{Br}$ & 5.90 \\
\hline \multirow[t]{3}{*}{ NPX } & 293.0 & $\mathrm{C}_{13} \mathrm{H}_{11} \mathrm{O}_{3} \mathrm{Br}$ & 4.88 \\
\hline & 265.0 & $\mathrm{C}_{12} \mathrm{H}_{11} \mathrm{O}_{2} \mathrm{Br}$ & 4.58 \\
\hline & 170.9 & $\mathrm{C}_{6} \mathrm{H}_{5} \mathrm{OBr}$ & 4.83 \\
\hline \multirow[t]{2}{*}{ PHE } & 250.9 & $\mathrm{C}_{6} \mathrm{H}_{4} \mathrm{OBr}_{2}$ & 5.76 \\
\hline & 328.8 & $\mathrm{C}_{6} \mathrm{H}_{3} \mathrm{OBr}_{3}$ & 6.58 \\
\hline
\end{tabular}


Table S6. Predicted acute and chronic toxicity a of parent compounds and their reaction products by $\mathrm{PAA} / \mathrm{Br}^{-}$process and general $\mathrm{Br}-\mathrm{DBPs}$ using the ECOSAR program.

\begin{tabular}{|c|c|c|c|c|c|c|}
\hline \multirow{2}{*}{ Compounds } & \multicolumn{3}{|c|}{ Acute toxicity $\left(\mathrm{mg} \cdot \mathrm{L}^{-1}\right)$} & \multicolumn{3}{|c|}{ Chronic toxicity $\left(\mathrm{mg} \cdot \mathrm{L}^{-1}\right): \mathrm{ChV}$} \\
\hline & $\begin{array}{l}\text { Fish } \\
\left(\mathrm{LC}_{50}\right)\end{array}$ & $\begin{array}{c}\text { Daphnid } \\
\left(\mathrm{LC}_{50}\right)\end{array}$ & $\begin{array}{c}\text { Green Algae } \\
\left(\mathrm{EC}_{50}\right)\end{array}$ & $\begin{array}{c}\text { Fish } \\
\left(\mathrm{LC}_{50}\right)\end{array}$ & $\begin{array}{c}\text { Daphnid } \\
\left(\mathrm{LC}_{50}\right)\end{array}$ & $\begin{array}{l}\text { Green Algae } \\
\left(\mathrm{EC}_{50}\right)\end{array}$ \\
\hline EE2 & 1.42 & 1.6 & & 0.17 & 0.25 & 0.47 \\
\hline $\mathrm{m} / \mathrm{z} 373.1^{\mathrm{b}}$ & 0.4 & 0.738 & 0.043 & 0.059 & 0.137 & 0.188 \\
\hline $\mathrm{m} / \mathrm{z} 389.1^{\mathrm{b}}$ & 0.65 & 1.88 & 1.15 & 0.246 & 0.605 & 0.223 \\
\hline $\mathrm{m} / \mathrm{z} 435.1$ & & & 0.0013 & & 0.016 & 0.0096 \\
\hline $\mathrm{m} / \mathrm{z} 451.0$ & & & & 0.019 & & 0.071 \\
\hline $\mathrm{m} / \mathrm{z} 469.0$ & 1.43 & 1.99 & & 0.04 & 0.101 & 0.587 \\
\hline $\mathrm{m} / \mathrm{z} 512.9$ & 0.0033 & 0 & 37 & 0.00063 & 0.0081 & 0.0035 \\
\hline $\mathrm{m} / \mathrm{z} 546.9$ & 0 & 0.339 & 53 & 0.059 & 0.102 & 0.15 \\
\hline SMX & 267 & 6.43 & 21.8 & 5.00 & 0.07 & 11.1 \\
\hline $\mathrm{m} / \mathrm{z} 332.0^{\mathrm{b}}$ & 91.8 & 4.99 & 14.5 & 1.18 & 0.06 & 5.11 \\
\hline NPX & 193 & 122 & 138 & 21.3 & 15.7 & 45.3 \\
\hline $\mathrm{m} / \mathrm{z} 307.0$ & 41.2 & 28.1 & 44.8 & 5.00 & 4.58 & 17.7 \\
\hline $\mathrm{m} / \mathrm{z} 293.0$ & 41.7 & 34.5 & 3.87 & 4.78 & 4.84 & 11.6 \\
\hline $\mathrm{m} / \mathrm{z} 265.0$ & 6.59 & 4.65 & 0.605 & 0.729 & 0.614 & 1.66 \\
\hline PHE & 27.7 & 9.64 & 2.40 & 2.61 & 0.97 & 4.53 \\
\hline $\mathrm{m} / \mathrm{z} 170.9$ & 12.46 & 6.47 & 1.11 & 1.29 & 0.76 & 2.60 \\
\hline $\mathrm{m} / \mathrm{z} 250.9$ & 4.43 & 3.44 & 041 & 0.5 & 0.47 & 1.18 \\
\hline $\mathrm{m} / \mathrm{z} 2328.8$ & 1.42 & 1.65 & 0.14 & 0.18 & 0.26 & 0.49 \\
\hline
\end{tabular}

a Toxicity was classified based on the Globally Harmonized System of Classification and Labeling of Chemicals. For acute toxicity: Very toxic (Red): LC50/EC50 $1 \mathrm{mg} \cdot \mathrm{L}^{-1}$, Toxic (Pink): $1 \mathrm{mg} \cdot \mathrm{L}^{-}$ $1<$ LC50/EC50 $\leq 10 \mathrm{mg} \cdot \mathrm{L}^{-1}$, Harmful (Yellow): $10 \mathrm{mg} \cdot \mathrm{L}^{-1}<\mathrm{LC} 50 / \mathrm{EC} 50 \leq 100 \mathrm{mg} \cdot \mathrm{L}^{-1}$, Not harmful (Green): LC50/EC50 $>100 \mathrm{mg} \cdot \mathrm{L}^{-1}$. For chronic toxicity: Very toxic (Red): LC50/EC50 $\leq 0.1$ $\mathrm{mg} \cdot \mathrm{L}^{-1}$, Toxic (Pink): $0.1 \mathrm{mg} \cdot \mathrm{L}^{-1}<\mathrm{LC} 50 / \mathrm{EC} 50 / \mathrm{ChV} \leq 1 \mathrm{mg} \cdot \mathrm{L}^{-1}$, Harmful (Yellow): $1 \mathrm{mg} \cdot \mathrm{L}^{-1}<$ LC50/EC50 $\leq 10 \mathrm{mg} \cdot \mathrm{L}^{-1}$, Not harmful (Green): LC50/EC50 > $10 \mathrm{mg} \cdot \mathrm{L}^{-1}$.

${ }^{b}$ In case the exact structure of the proposed product is not clear, the highest toxicity from possible structures is indicated. 


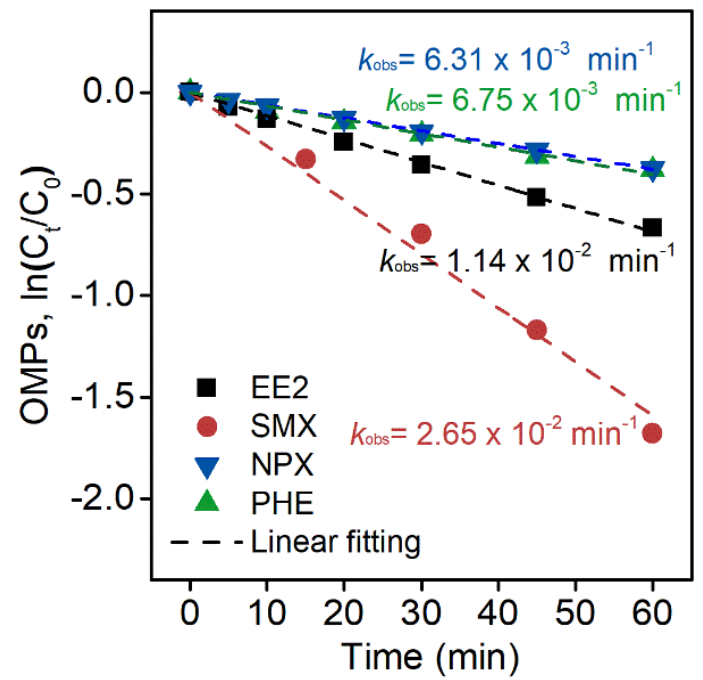

Figure S1. Linear fitting of selected OMPs degradation by the $\mathrm{PAA} / \mathrm{Br}^{-}$process. Experimental conditions: $\mathrm{pH} 7.1,[\mathrm{OMPs}]_{0}=10 \mu \mathrm{M},[\mathrm{PAA}]_{0}=0.2 \mathrm{mM},\left[\mathrm{Br}^{-}\right]_{0}=0.2 \mathrm{mM}$, and room temperature $\left(22 \pm 2{ }^{\circ} \mathrm{C}\right)$. Error bars of each data point indicate the standard deviation of duplicates. 


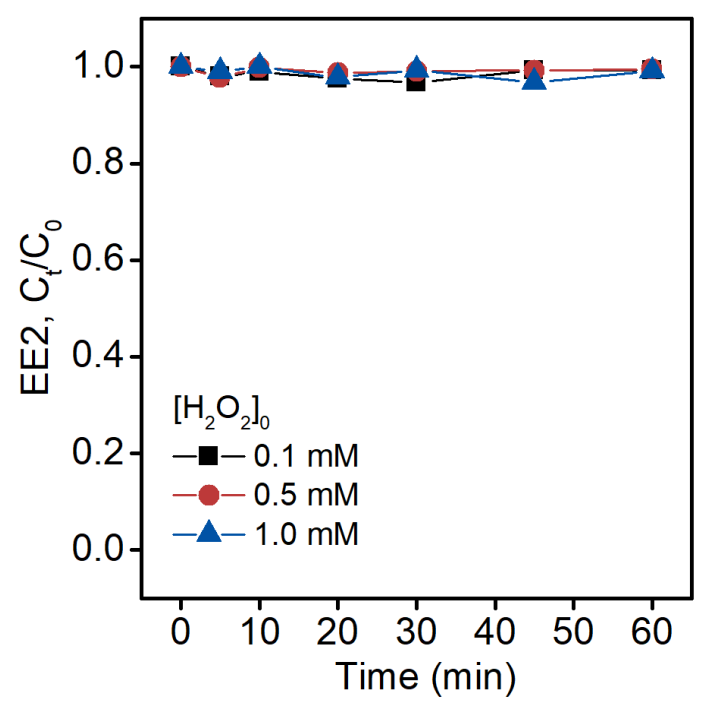

Figure S2. Influence of initial $\mathrm{H}_{2} \mathrm{O}_{2}$ concentration on EE2 degradation by the $\mathrm{H}_{2} \mathrm{O}_{2} / \mathrm{Br}^{-}$process. Experimental conditions: $\mathrm{pH} 7.0,[\mathrm{EE} 2]_{0}=10 \mu \mathrm{M},\left[\mathrm{Br}^{-}\right]_{0}=0.2 \mathrm{mM}$, and room temperature $(22 \pm$ $\left.2{ }^{\circ} \mathrm{C}\right)$. 


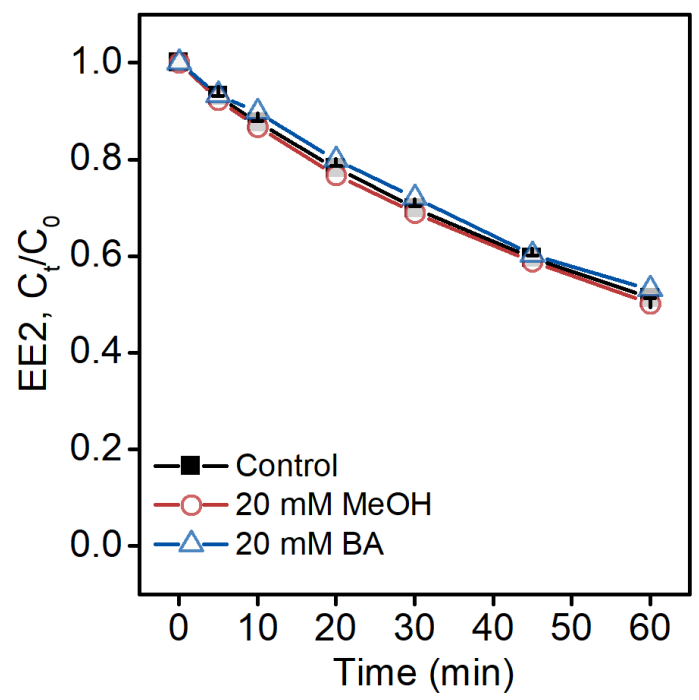

Figure S3. Influence of radical scavengers $\mathrm{MeOH}$ and BA on EE2 degradation by the PAA/Br process. Experimental conditions: $\mathrm{pH} 7.0,[\mathrm{EE} 2]_{0}=10 \mu \mathrm{M},[\mathrm{PAA}]_{0}=0.2 \mathrm{mM},\left[\mathrm{Br}^{-}\right]_{0}=0.2 \mathrm{mM}$, and room temperature $\left(22 \pm 2{ }^{\circ} \mathrm{C}\right)$. 


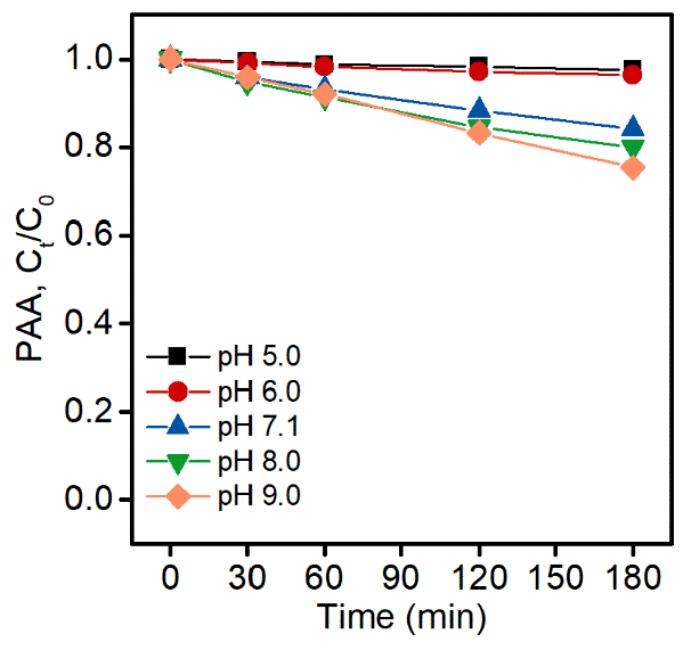

Figure S4. Effect of $\mathrm{pH}$ on PAA spontaneous decay. Experimental conditions: $[\mathrm{PAA}]_{0}=30 \mu \mathrm{M}$, and room temperature $\left(22 \pm 2{ }^{\circ} \mathrm{C}\right)$. 


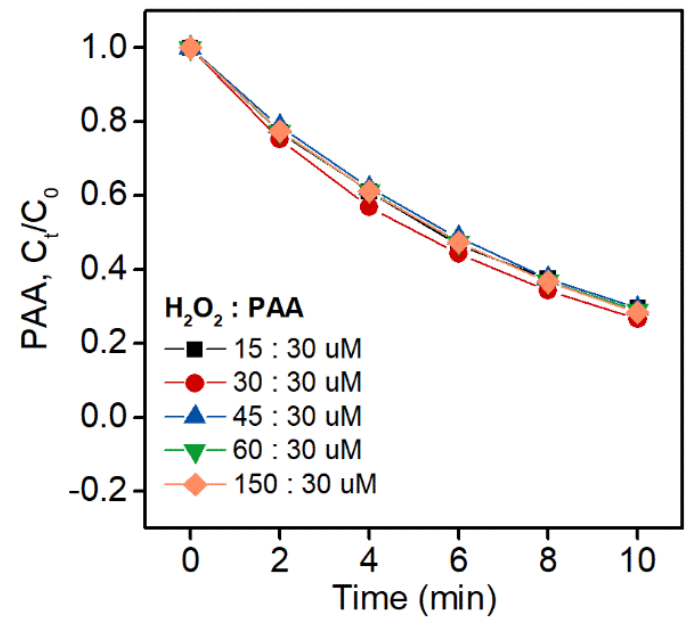

Figure S5. Effect of $\mathrm{H}_{2} \mathrm{O}_{2}$ on PAA decay. Experimental conditions: $\mathrm{pH} 7.0$, $[\mathrm{PAA}]_{0}=30 \mu \mathrm{M}$, $\left[\mathrm{Br}^{-}\right]_{0}=15 \mathrm{mM}$, and room temperature $\left(22 \pm 2{ }^{\circ} \mathrm{C}\right)$. 


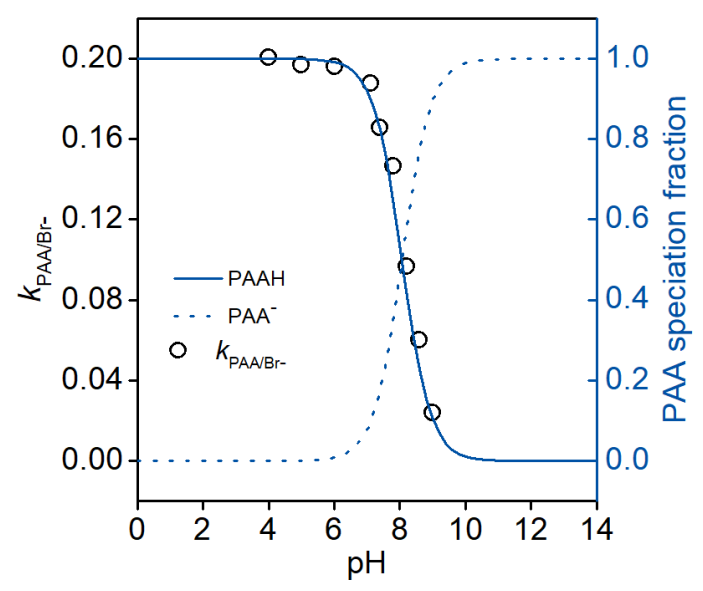

Figure S6. $\mathrm{pH}$-dependence of $k_{\mathrm{PAA} / \mathrm{Br}^{-}}$with an $\mathrm{pH}$ range of 4.0-9.0 as well as the PAA speciation distribution. 


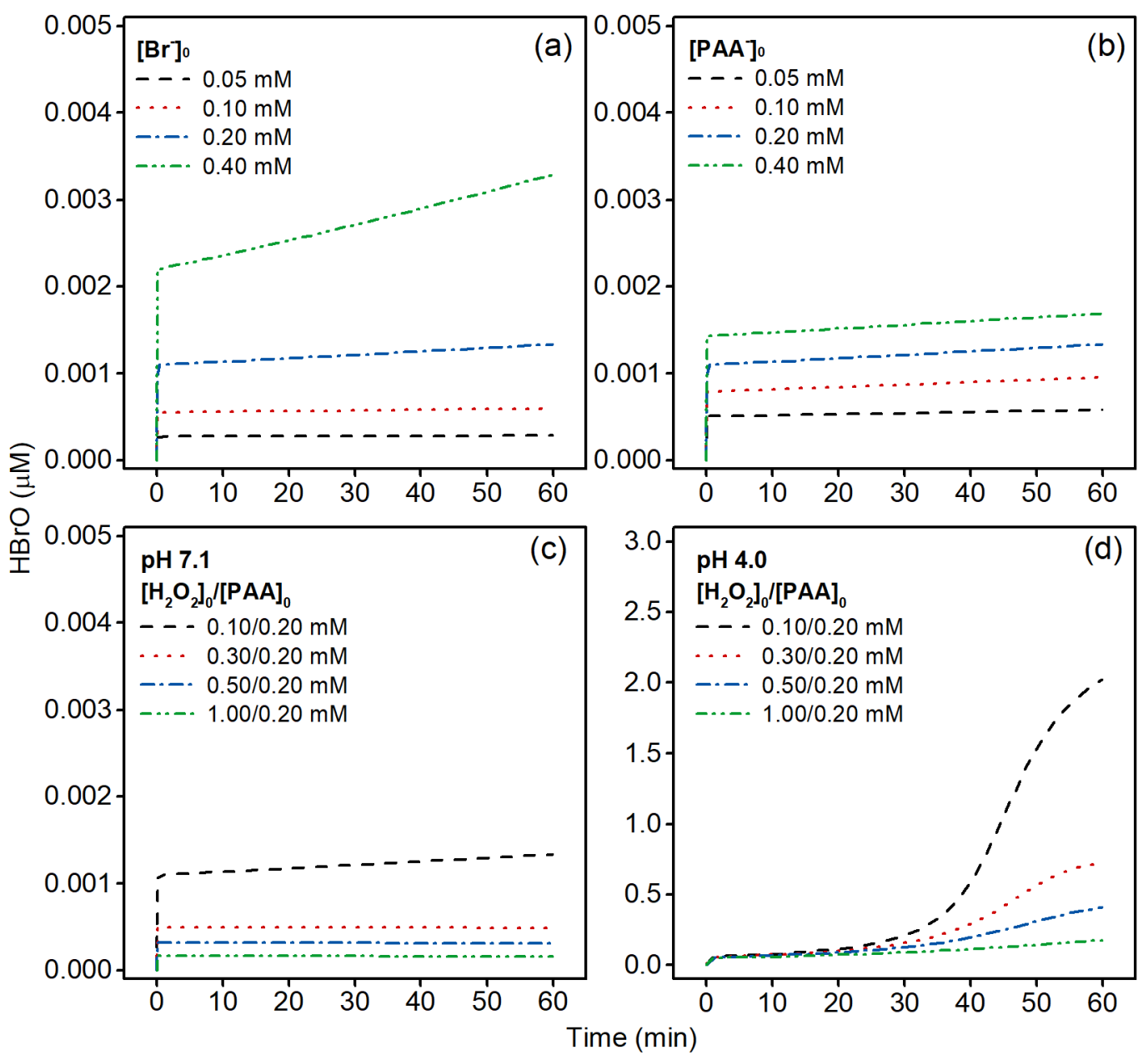

Figure S7. Simulated HBrO formation during EE2 degradation at different (a) $\left[\mathrm{Br}^{-}\right]_{0}(0.05-0.40$ $\mathrm{mM})$; (b) $[\mathrm{PAA}]_{0}(0.05-0.40 \mathrm{mM})$; (c) $\left[\mathrm{H}_{2} \mathrm{O}_{2}\right]_{0}(0.10-1.00 \mathrm{mM})$ at $\mathrm{pH} 7.1$; (d) $\left[\mathrm{H}_{2} \mathrm{O}_{2}\right]_{0}(0.10-1.00$ $\mathrm{mM})$ at $\mathrm{pH} 4.0$. For all reactions: $[\mathrm{EE} 2]_{0}=10 \mu \mathrm{M},\left[\mathrm{Br}^{-}\right]_{0}=0.20 \mathrm{mM}$ except for (a), $[\mathrm{PAA}]_{0}=0.20$ $\mathrm{mM}$ except for (b), $\left[\mathrm{H}_{2} \mathrm{O}_{2}\right]_{0}=0.10 \mathrm{mM}$ except for (c) and (d), $\mathrm{pH}=7.1$ except for (d). 


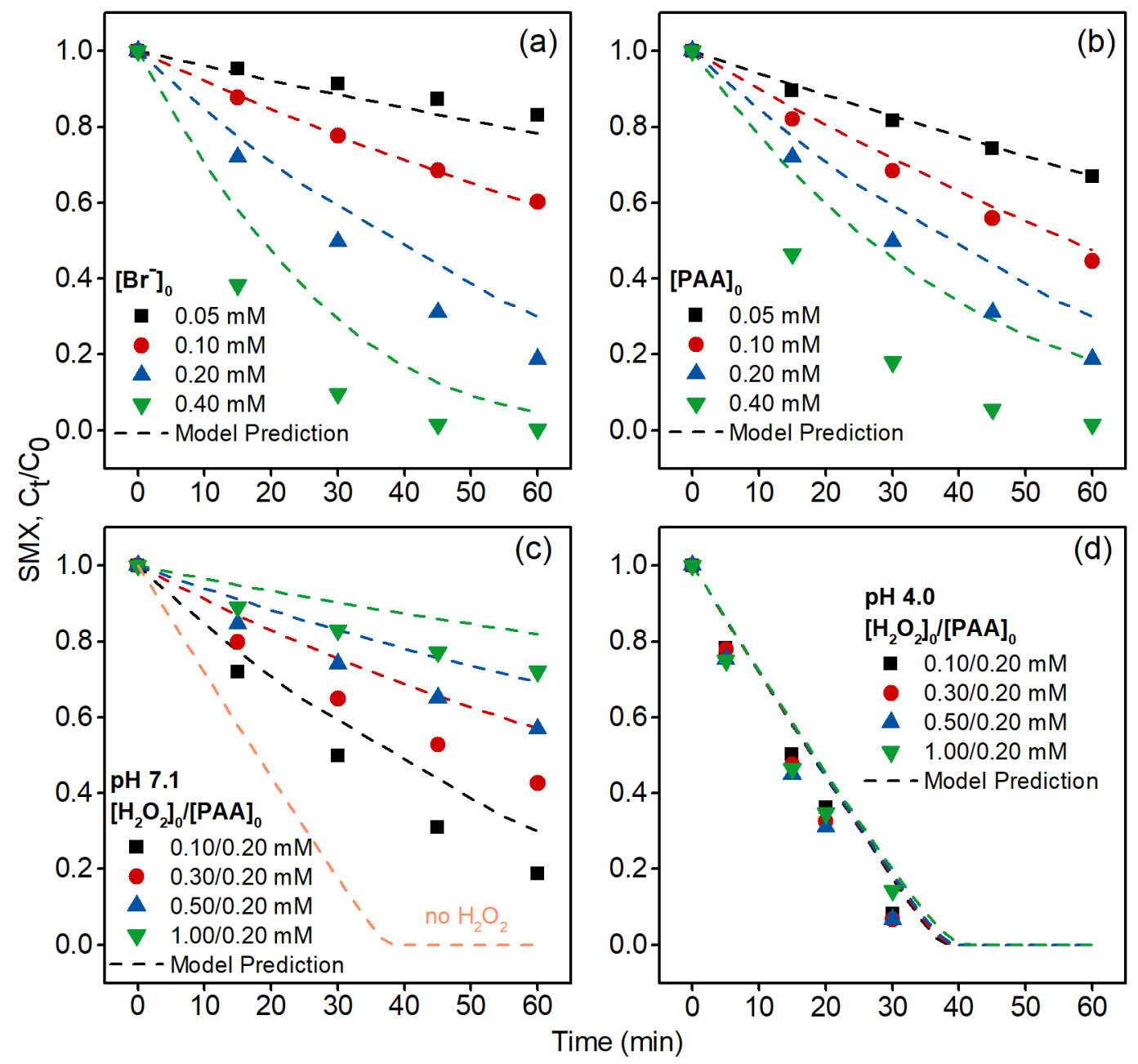

Figure S8. Degradation of SMX by the PAA/ $\mathrm{Br}^{-}$system at different (a) $\left[\mathrm{Br}^{-}\right]_{0}(0.05-0.40 \mathrm{mM})$; (b) $[\mathrm{PAA}]_{0}(0.05-0.40 \mathrm{mM}) ;$ (c) $\left[\mathrm{H}_{2} \mathrm{O}_{2}\right]_{0}(0.10-1.00 \mathrm{mM})$ at $\mathrm{pH} 7.1 ;(\mathrm{d})\left[\mathrm{H}_{2} \mathrm{O}_{2}\right]_{0}(0.10-1.00 \mathrm{mM})$ at $\mathrm{pH}$ 4.0. For all reactions: $[\mathrm{SMX}]_{0}=10 \mu \mathrm{M},\left[\mathrm{Br}^{-}\right]_{0}=0.20 \mathrm{mM}$ except for (a), $[\mathrm{PAA}]_{0}=0.20$ $\mathrm{mM}$ except for (b), $\left[\mathrm{H}_{2} \mathrm{O}_{2}\right]_{0}=0.10 \mathrm{mM}$ except for (c) and (d), $\mathrm{pH}=7.1$ except for (d). Dashed lines represent model predictions by considering reactions in Table 1 and S3. 


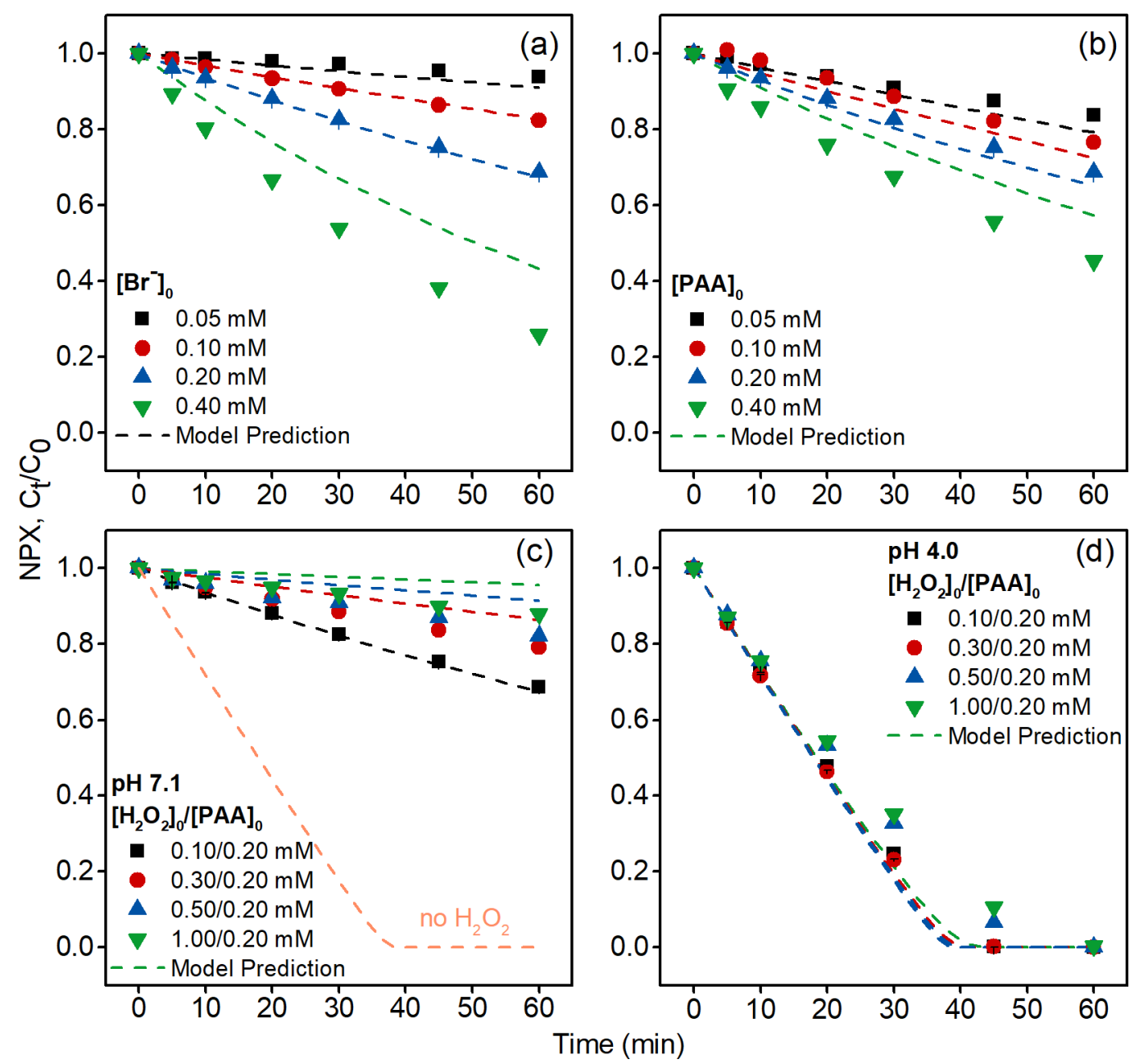

Figure S9. Degradation of NPX by the PAA/ $\mathrm{Br}^{-}$system at different (a) $\left[\mathrm{Br}^{-}\right]_{0}(0.05-0.40 \mathrm{mM})$; (b) $[\mathrm{PAA}]_{0}(0.05-0.40 \mathrm{mM})$; (c) $\left[\mathrm{H}_{2} \mathrm{O}_{2}\right]_{0}(0.10-1.00 \mathrm{mM})$ at $\mathrm{pH} 7.1 ;(\mathrm{d})\left[\mathrm{H}_{2} \mathrm{O}_{2}\right]_{0}(0.10-1.00 \mathrm{mM})$ at $\mathrm{pH}$ 4.0. For all reactions: $[\mathrm{NPX}]_{0}=10 \mu \mathrm{M},\left[\mathrm{Br}^{-}\right]_{0}=0.20 \mathrm{mM}$ except for (a), $[\mathrm{PAA}]_{0}=0.20$ $\mathrm{mM}$ except for (b), $\left[\mathrm{H}_{2} \mathrm{O}_{2}\right]_{0}=0.10 \mathrm{mM}$ except for (c) and (d), $\mathrm{pH}=7.1$ except for (d). Dashed lines represent model predictions by considering reactions in Table 1 and S3. 


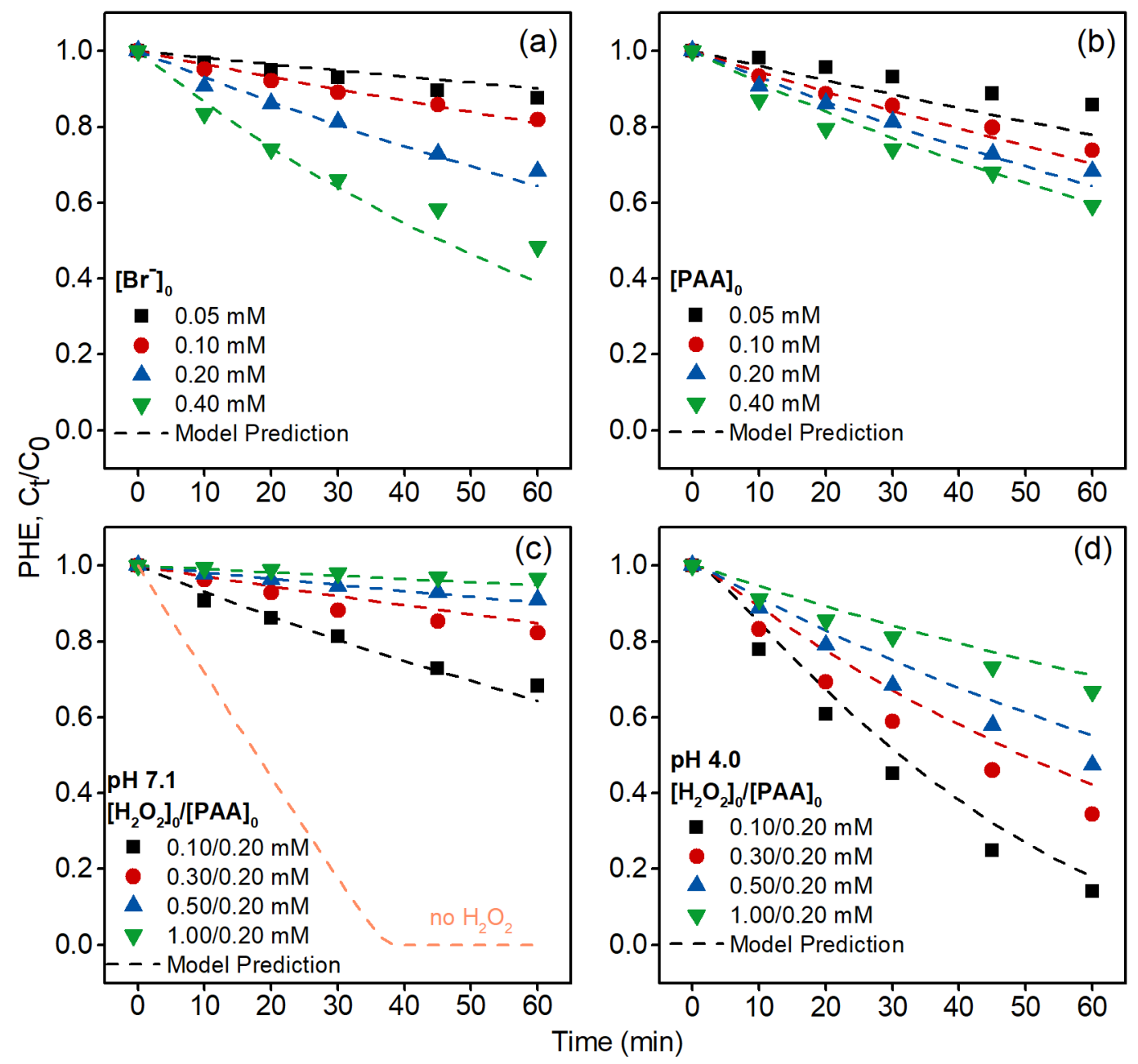

Figure S10. Degradation of PHE by the PAA/ Br ${ }^{-}$process at different (a) $\left[\mathrm{Br}^{-}\right]_{0}(0.05-0.40$ $\mathrm{mM})$; (b) $[\mathrm{PAA}]_{0}(0.05-0.40 \mathrm{mM})$; (c) $\left[\mathrm{H}_{2} \mathrm{O}_{2}\right]_{0}(0.10-1.00 \mathrm{mM})$ at $\mathrm{pH} 7.1$; (d) $\left[\mathrm{H}_{2} \mathrm{O}_{2}\right]_{0}(0.10-$ $1.00 \mathrm{mM}$ ) at $\mathrm{pH} 4.0$. For all reactions: $[\mathrm{PHE}]_{0}=10 \mu \mathrm{M},\left[\mathrm{Br}^{-}\right]_{0}=0.20 \mathrm{mM}$ except for (a), [PAA $]_{0}$ $=0.20 \mathrm{mM}$ except for (b), $\left[\mathrm{H}_{2} \mathrm{O}_{2}\right]_{0}=0.10 \mathrm{mM}$ except for (c) and (d), $\mathrm{pH}=7.1$ except for (d). Dashed lines represent model predictions by considering reactions in Table 1 and S3. 


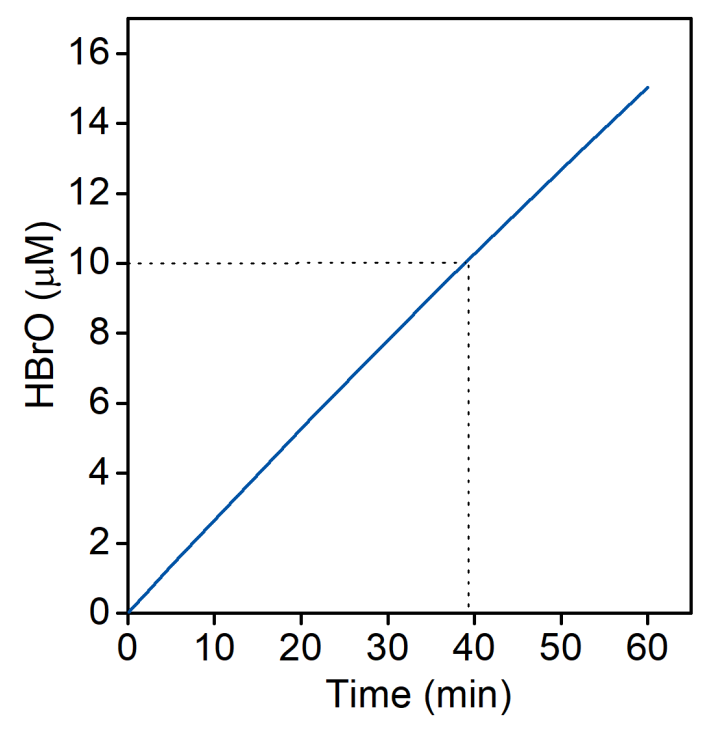

Figure S11. Simulated $\mathrm{HBrO}$ formation in the $\mathrm{PAA} / \mathrm{Br}^{-}$system. Experimental condition: $\left[\mathrm{Br}^{-}\right]_{0}=$ $0.20 \mathrm{mM},[\mathrm{PAA}]_{0}=0.20 \mathrm{mM},\left[\mathrm{H}_{2} \mathrm{O}_{2}\right]_{0}=0.10 \mathrm{mM}, \mathrm{pH}=4.0$, and room temperature $\left(22 \pm 2{ }^{\circ} \mathrm{C}\right)$. 

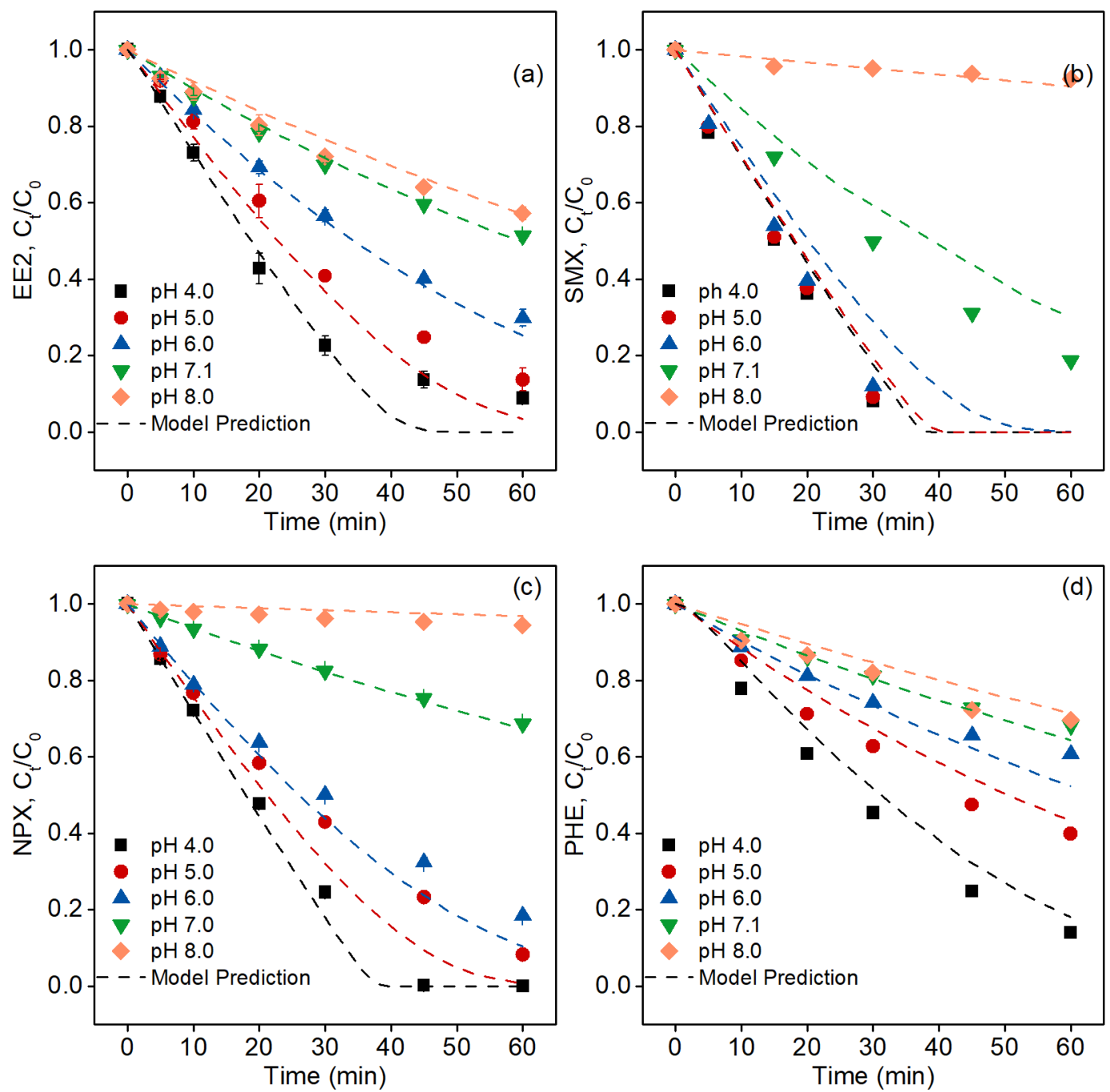

Figure S12. Effect of the pH on the EE2(a), SMX(b), NPX(c), and PHE(d) degradation by the PAA $/ \mathrm{Br}^{-}$process. Experimental conditions: $[\mathrm{OMP}]_{0}=10 \mu \mathrm{M},\left[\mathrm{Br}^{-}\right]_{0}=0.20 \mathrm{mM},[\mathrm{PAA}]_{0}=0.20$ $\mathrm{mM}$, and room temperature $\left(22 \pm 2{ }^{\circ} \mathrm{C}\right)$. Dashed lines represent model predictions by considering reactions in Table 1 and $\mathrm{S} 3$. 


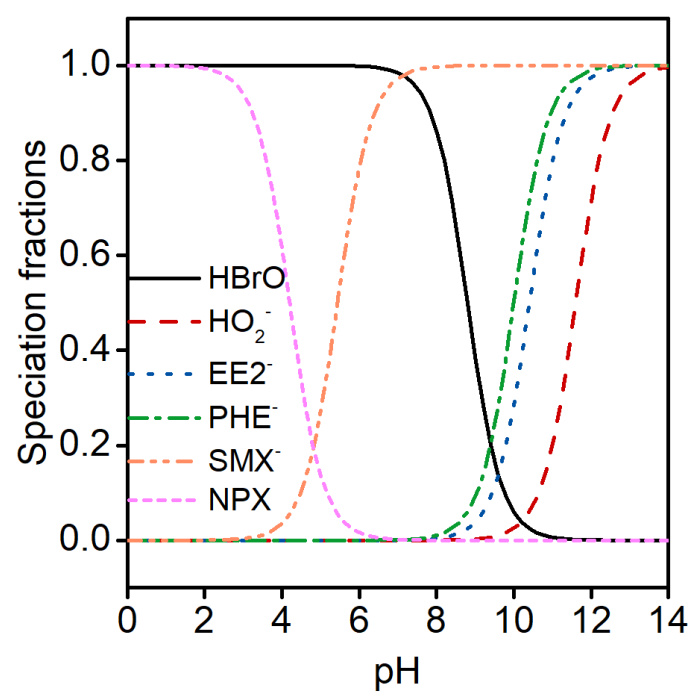

Figure S13. Speciation fraction distribution of primary reactive species involved in the studied $\mathrm{PAA} / \mathrm{Br}^{-} / \mathrm{OMPs}$ systems. 

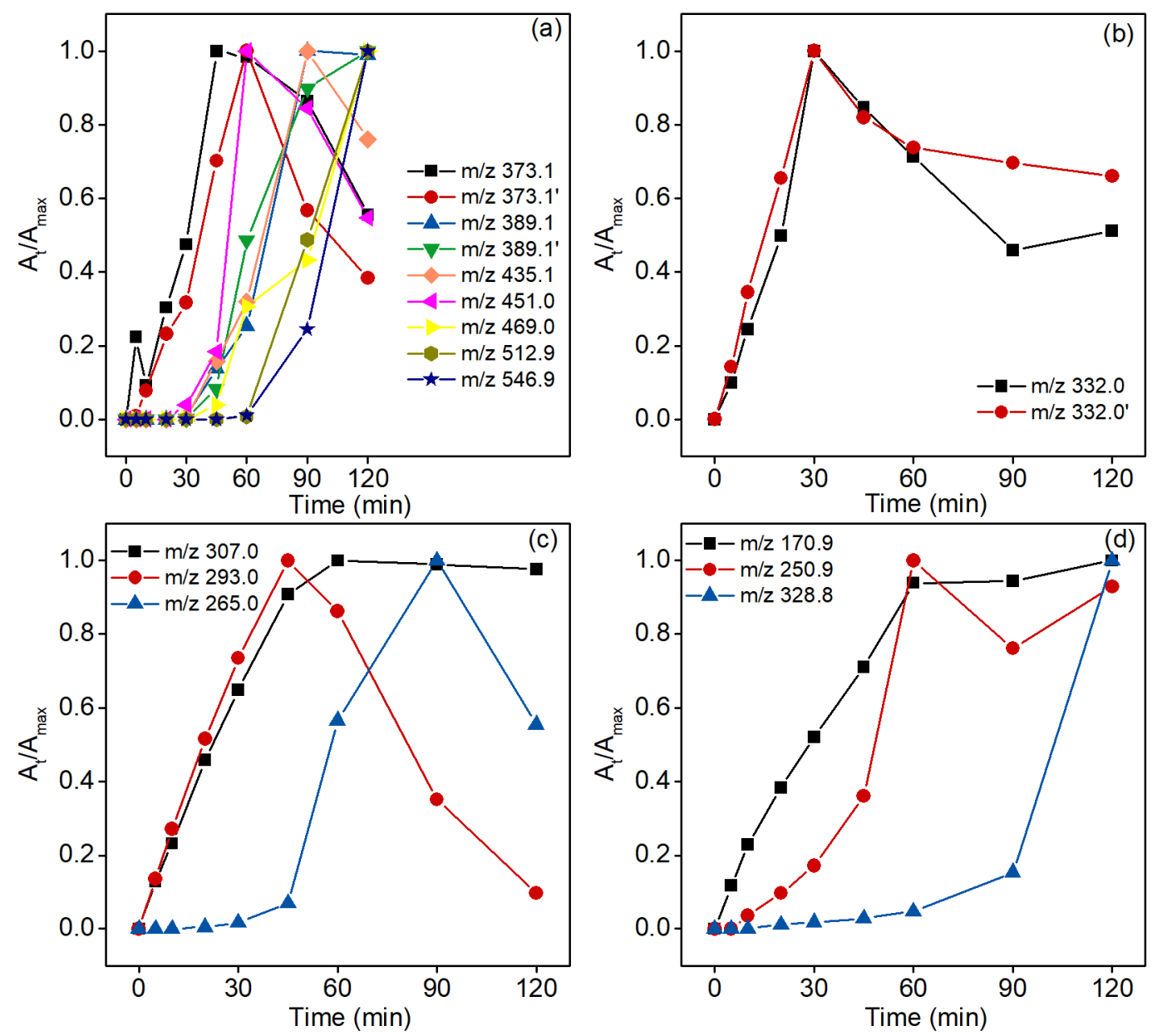

Figure S14. Reaction products of (a) EE2, (b) SMX, (c) NPX, and (d) PHE in the PAA/Br- process with different reaction times. Reaction condition: $[\mathrm{OMP}]_{0}=10 \mu \mathrm{M},[\mathrm{PAA}]_{0}=0.2 \mathrm{mM},\left[\mathrm{Br}^{-}\right]_{0}=$ $0.2 \mathrm{mM}, \mathrm{pH} 4.0$, and room temperature $\left(22 \pm 2^{\circ} \mathrm{C}\right)$. 


\section{References:}

1. USEPA ECOSAR (Elogical Structure Activity Relationships) v.2.0. https://www.epa.gov /tsca-screening-tools/ecological-structureactivity-relationships-ecosar-predictive-model (accessed October 11, 2020).

2. Heeb, M. B.; Criquet, J.; Zimmermann-Steffens, S. G.; von Gunten, U., Oxidative treatment of bromide-containing waters: Formation of bromine and its reactions with inorganic and organic compounds - A critical review. Water Res. 2014, 48, 15-42.

3. Dodd, M. C.; Huang, C.-H., Transformation of the antibacterial agent sulfamethoxazole in reactions with chlorine: Kinetics, mechanisms, and pathways. Environ. Sci. Technol. 2004, $38,(21), 5607-5615$.

4. Benitez, F. J.; Acero, J. L.; Real, F. J.; Roldan, G.; Casas, F., Bromination of selected pharmaceuticals in water matrices. Chemosphere 2011, 85, (9), 1430-1437.

5. Acero, J. L.; Piriou, P.; von Gunten, U., Kinetics and mechanisms of formation of bromophenols during drinking water chlorination: Assessment of taste and odor development.

Water Res. 2005, 39, (13), 2979-2993.

6. Gallard, H.; Pellizzari, F.; Croue, J. P.; Legube, B., Rate constants of reactions of bromine with phenols in aqueous solution. Water Res. 2003, 37, (12), 2883-2892.

7. Guo, G.; Lin, F., The bromination kinetics of phenolic compounds in aqueous solution. $J$. Hazard. Mater. 2009, 170, (2), 645-651. 